\title{
Localization Transition of the Three-Dimensional Lorentz Model and Continuum Percolation
}

\author{
Felix Höfling, ${ }^{1}$ Thomas Franosch, ${ }^{1,2}$ and Erwin Frey ${ }^{2}$ \\ ${ }^{1}$ Hahn-Meitner-Institut Berlin, Abteilung Theorie, Glienicker Straße 100, D-14109 Berlin, Germany \\ ${ }^{2}$ Arnold Sommerfeld Center and CeNS, Department of Physics, Ludwig-Maximilians-Universität München,
} Theresienstraße 37, D-80333 München, Germany

(Received 17 October 2005; published 24 April 2006)

\begin{abstract}
The localization transition and the critical properties of the Lorentz model in three dimensions are investigated by computer simulations. We give a coherent and quantitative explanation of the dynamics in terms of continuum percolation theory and obtain an excellent matching of the critical density and exponents. Within a dynamic scaling ansatz incorporating two divergent length scales we achieve data collapse for the mean-square displacements and identify the leading corrections to scaling. We provide evidence for a divergent non-Gaussian parameter close to the transition.
\end{abstract}

DOI: 10.1103/PhysRevLett.96.165901

Transport in heterogeneous and disordered media has important applications in many fields of science including composite materials, rheology, polymer and colloidal science, and biophysics. Recently, dynamic heterogeneities and growing cooperative length scales in structural glasses have attracted considerable interest [1,2]. The physics of gelation, in particular, of colloidal particles with short range attraction [3-6], is often accompanied by the presence of a fractal cluster generating subdiffusive dynamics. It is of fundamental interest to demonstrate the relevance of such heterogeneous environments on slow anomalous transport.

The minimal model for transport of particles through a random medium of fixed obstacles is known as the Lorentz model, and already incorporates the generic ingredients for slow anomalous transport. Earlier, the Lorentz model played a significant role as a testing ground for elaborate kinetic theories, shortly after the discovery of long-time tails in autocorrelation functions for simple liquids in the late 1960s [7], since the nonanalytic dependence of transport coefficients on frequency, wave number, and density predicted for simple liquids [8-12] has a close analog in the Lorentz model $[13,14]$.

The simplest variant of the Lorentz model consists of a structureless test particle moving according to Newton's laws in a $d$-dimensional array of identical obstacles. The latter are distributed randomly and independently in space and interact with the test particle via a hard-sphere repulsion. Consequently, the test particle explores a disordered environment of possibly overlapping regions of excluded volume; see Fig. 1. Because of the hard-core repulsion, the magnitude of the particle velocity, $v=|\mathbf{v}|$, is conserved. Then, the only control parameter is the dimensionless obstacle density, $n^{*}:=n \sigma^{d}$, where $\sigma$ denotes the radius of the hard-core potential. At high densities, the model exhibits a localization transition, i.e., above a critical density, the particle is always trapped by the obstacles.

Significant insight into the dynamic properties of the Lorentz model has been achieved by a low-density expansion for the diffusion coefficient by Weijland and
PACS numbers: 66.30.Hs, 05.40.- a, 61.43. $-\mathrm{j}, 64.60 . \mathrm{Ht}$

van Leeuwen [13] rigorously demonstrating the nonanalytic dependence on $n^{*}$. As expected, for low densities the theoretical results compare well with molecular dynamics (MD) simulations [15]. Elaborate self-consistent kinetic theories $[16,17]$ have allowed going much beyond such perturbative approaches. They give a mathematically consistent description of the localization transition, which allows the calculation of the critical density within a $20 \%$ accuracy and allows the extension of the regime of quantitative agreement to intermediate densities. In addition, they have provided a microscopic approach towards anomalous transport and mean-field-like scaling behavior [16].

A different line of approach focusing on the localization transition starts from the fractal nature of the void space between the overlapping spheres in the Lorentz model and considers it as a continuum percolation problem [18-22],

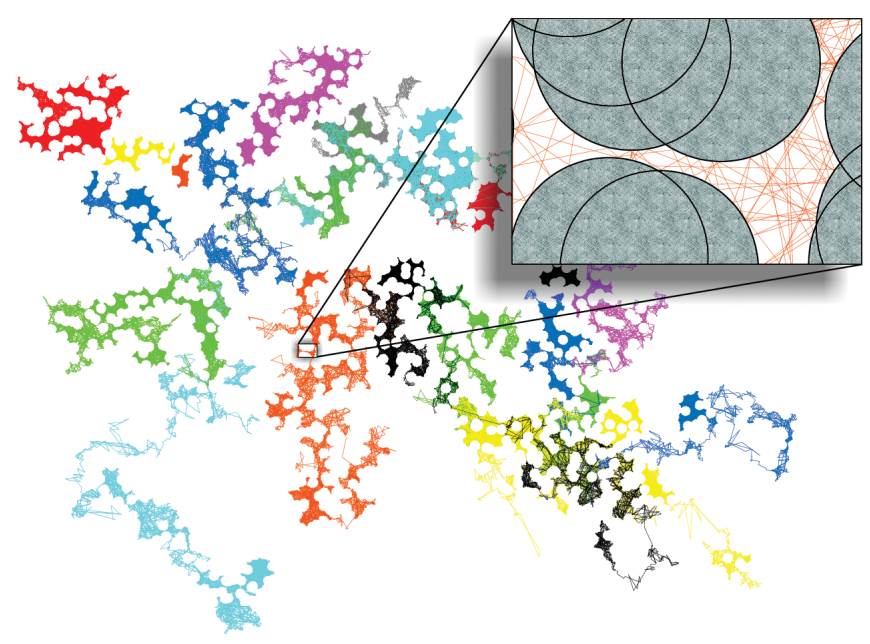

FIG. 1 (color). Typical particle trajectories in a 2D Lorentz model slightly below $n_{c}^{*}$ over a few thousand collisions each. Colors encode different initial conditions; obstacles have been omitted for clarity. Most trajectories being in the percolating void space have some overlap; a few trajectories are confined to finite clusters. Blowup: a particle squeezes through narrow gaps formed by the obstacles. 
which in this context has also been termed the "Swiss cheese" model [20]. These authors conjectured that the transport properties close to the percolation threshold can be obtained by analyzing an equivalent random resistor network. The equivalence, however, has been shown only for geometric properties close to the percolation point [23]. As a peculiarity of continuum percolation, differences to lattice percolation may arise due to power-law tails in the probability distribution of the conductances ("narrow gaps"). Such random resistor networks have been investigated extensively by means of Monte Carlo simulations $[24,25]$ and renormalization group techniques [26,27], providing reliable numeric and analytic results for the critical behavior [28].

In this Letter, we present a direct numerical analysis of the dynamic properties of the Lorentz model without resorting to random resistor networks. By means of extensive MD simulations, we obtain a quantitative description of the dynamic properties over the full density range, in particular, focusing on both sides of the critical region. This allows for a quantitative test of the conjectured mappings to continuum percolation theory. Furthermore, we explore the range of validity of the dynamic scaling hypothesis for the Lorentz model [29]. The probability distribution of particle displacements, i.e., the van Hove self-correlation function, $G(\mathbf{r}, t):=\langle\delta(\mathbf{r}-\Delta \mathbf{R}(t))\rangle$, and its second moment, the mean-square displacement (MSD), $\delta r^{2}(t):=$ $\left\langle|\Delta \mathbf{R}(t)|^{2}\right\rangle$, are the appropriate quantities for this purpose; $\Delta \mathbf{R}(t)=\mathbf{R}(t)-\mathbf{R}(0)$ denotes the displacement of the test particle at time $t$.

Over a wide range of obstacle densities, we have simulated several hundred trajectories in three dimensions, employing an event-oriented MD algorithm. For each of $N_{r}$ different realizations of the obstacle disorder, a set of $N_{t}$ trajectories with different initial conditions is simulated. Below the critical density, we have chosen $N_{r} \geq 25$ and $N_{t} \geq 4$. At very high densities, where the phase space is highly decomposed, these values have been increased up to $N_{r} \times N_{t}=600$. In order to minimize finite-size effects, the size of the simulation box, $L_{\mathrm{box}}$, has been chosen significantly larger than the correlation length $\xi, L_{\mathrm{box}}=$ $200 \sigma \gg \xi[30]$.

The results for the MSD cover a nontrivial time window of more than seven decades for densities close to the transition, see Fig. 2(a). At low densities, one observes only a trivial crossover from ballistic motion, $\delta r^{2}(t)=$ $v^{2} t^{2}$, to diffusion, $\delta r^{2}(t) \sim t$, near the mean collision time $\tau=1 / \pi n v \sigma^{2}$ as expected from Boltzmann theory. With increasing density, an intermediate time window opens where motion becomes subdiffusive, $\delta r^{2}(t) \sim t^{2 / z}$ with $z>2$. This time window extends to larger and larger times upon approaching a certain critical density $n_{c}^{*}$. For the density $n^{*}=0.84$, the subdiffusive behavior is obeyed over more than five decades and is compatible with a value of $z \approx 6.25$. The power law, $\delta r^{2}(t) \sim t^{2 / z}$, indicated in Fig. 2(a), nicely discriminates trajectories above and below
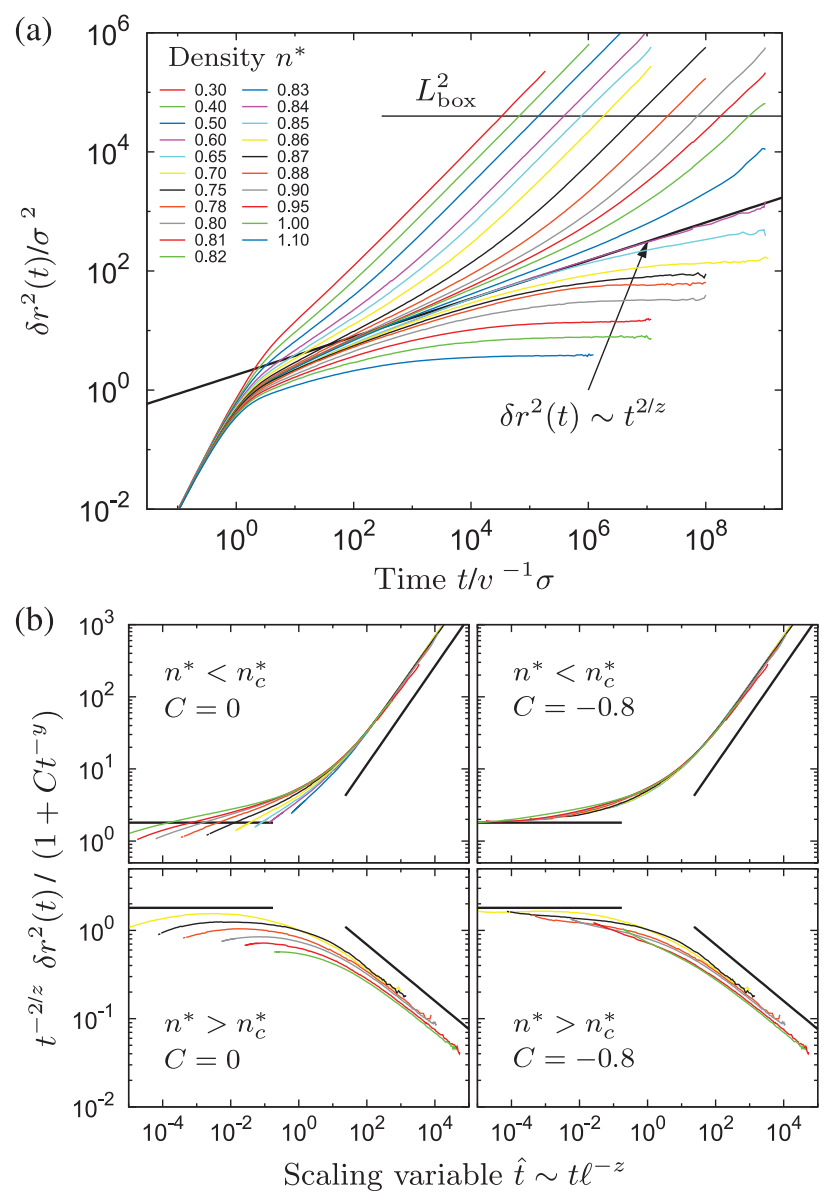

FIG. 2 (color). (a) MSD $\delta r^{2}(t)$ for various obstacle densities $n^{*}$ varying from 0.30 (top) to 1.10 (bottom). The thick black line represents a power law, $\delta r^{2}(t) \sim t^{2 / z}$ with $z=6.25$. (b) Scaling functions $\delta \hat{r}_{ \pm}^{2}(\hat{t})$ for the MSD. Right panels include corrections to scaling at leading order. Units are chosen such that $v=\sigma=$ 1; color key as in Fig. 2(a).

$n_{c}^{*}$. One also observes a density-dependent length scale $l$ characterizing the end of the subdiffusive regime by $\delta r^{2}(t) \simeq l^{2}$; upon approaching $n_{c}^{*}$ this crossover length scale $l$ is found to diverge. For long times, the dynamics eventually becomes either diffusive or localized for densities below or above $n_{c}^{*}$, respectively.

The diffusion coefficient $D$ has been extracted from the long-time limit of $\delta r^{2}(t) / 6 t$; in Fig. 3, $D$ is shown in units of the Boltzmann result, $D_{0}=\tau v^{2} / 3$. With increasing density, $D$ is more and more suppressed until it vanishes at $n_{c}^{*}$ as a power law, $D \sim|\varepsilon|^{\mu}$, where $\varepsilon:=\left(n^{*}-n_{c}^{*}\right) / n_{c}^{*}$ defines the separation parameter. Anticipating the exponent $\mu$ from percolation theory, a fit to our data yields the critical density, $n_{c}^{*}=0.839(4)$ [31], and the power-law behavior is confirmed over five decades in $D$. Above the critical density, the long-time limit of the MSD is compatible with a power law over more than one decade, $l \sim$ $\varepsilon^{-\nu+\beta / 2}$, where $\nu-\beta / 2 \approx 0.68$ (bottom inset in Fig. 3). Our finding of $n_{c}^{*}$ coincides with the percolation point of the void space $[18,19,32]$. This provides clear evidence for the intimate connection between continuum percolation 
and the Lorentz model; i.e., diffusion is not blocked as long as there is an infinite path through the medium - a purely geometric reason.

Considering the underlying continuum percolation problem, a geometric transition occurs at $n_{c}^{*}$, above which the void space falls completely apart into finite clusters. Just below this density, the volume fraction $P$ of the percolating void space (infinite cluster) vanishes as a power law, $P \sim$ $|\varepsilon|^{\beta}$. There are two divergent length scales characterizing the structure of the percolation network: the linear dimension of the largest finite clusters, $\xi \sim|\varepsilon|^{-\nu}$, and the mean cluster radius (radius of gyration), $l \sim|\varepsilon|^{-\nu+\beta / 2}$ [33]. The geometric exponents $\beta$ and $\nu$ are believed to be the same for lattice and continuum percolation [19]. Our results in Fig. 3 clearly identify the geometric mean cluster radius $l$ with the localization length of the MSD as anticipated by our choice of notation.

In continuum percolation, transport of a particle is limited by narrow gaps in the void space. It was argued that this feature of the dynamics is captured by an associated random resistor network with a distribution $\rho(W)$ of weak conductances $W$ exhibiting a power-law tail, $\rho(W) \sim$ $W^{-\alpha}, \alpha<1$ for small $W[20,21]$. Depending on the value of $\alpha$, the suppression of diffusion, $D \sim|\varepsilon|^{\mu}$, may be dominated by this tail, and dynamic exponents become different from lattice percolation, $\mu>\mu^{\text {lat }}$. In this case, the hyperscaling relation, $\mu=(d-2) \nu+1 /(1-\alpha)$, holds $[22,34]$. There is a discrepancy in the literature about the value of $\alpha$ in the Lorentz model [20,21,28]. Only the result of Machta and Moore [21], $\alpha=(d-2) /(d-1)$, is consistent with our data. In $d=3$, it implies $\mu=\nu+2 \approx$ 2.88 , and therefore, $\mu>\mu^{\text {lat }} \approx 2.0$ [35]. By means of a scaling relation [33], $z=(2 \nu-\beta+\mu) /(\nu-\beta / 2)$, one finds the dynamic exponent, $z \approx 6.25$, describing anomalous transport at critical density, $\delta r^{2}(t) \sim t^{2 / z}$. Note that this dynamic exponent is not independent but entirely

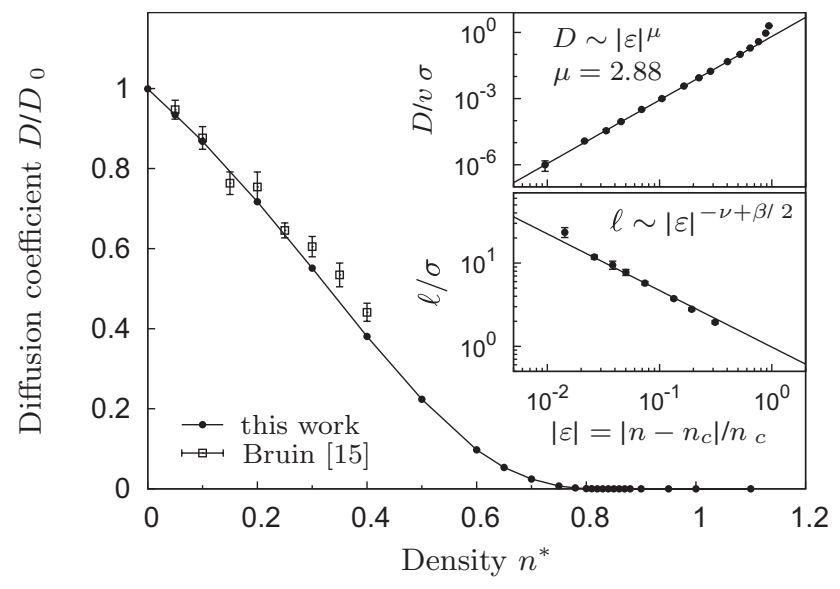

FIG. 3. Suppression of the diffusion coefficient $D / D_{0}$ with increasing density $n^{*}$. Top inset: Power-law behavior of $D$ close to $n_{c}^{*}$. Bottom inset: Divergence of the localization length $l$ upon approaching $n_{c}^{*}$ with exponent $\nu-\beta / 2=0.68$. determined by the geometric properties of the random environment.

In conclusion, the values obtained from the simulated MSD for the critical density $n_{c}^{*}$, the dynamic exponent $z$ as well as the exponents for the diffusion coefficient $\mu$ and the localization length $\nu-\beta / 2$ agree with the predicted values for continuum percolation. Within the statistical accuracy, no deviations can be inferred.

The quality of our data allows to go beyond determining critical exponents and to give a full analysis of the dynamic scaling properties. It has been argued by Kertész and Metzger [29] that the van Hove correlation function obeys scaling. Rewriting their ansatz in a more transparent way yields,

$$
G(\mathbf{r}, t ; \varepsilon)=\xi^{-\beta / \nu-d} G_{ \pm}\left(\mathbf{r} / \xi, t l^{-z}\right),
$$

where $G_{ \pm}$are master functions above (+) and below (-) the critical density. This ansatz clearly reflects the role of the two length scales: the correlation length $\xi$ rescales geometry whereas the crossover length scale $l$ rescales time. The scaling form of the MSD is easily inferred from $\delta r^{2}(t ; \varepsilon)=\int d^{d} \mathbf{r} r^{2} G(\mathbf{r}, t ; \varepsilon) \quad$ as, $\quad \delta r^{2}(t ; \varepsilon)=t^{2 / z} \delta \hat{r}_{ \pm}^{2}(\hat{t})$, where $\hat{t} \sim t l^{-z}$. Plotting $t^{-2 / z} \delta r^{2}(t ; \varepsilon)$ versus $\hat{t}$ for various densities [left panels of Fig. 2(b)], the data collapse nicely in the diffusive and localized regimes $(\hat{t} \gg 1)$ and converge rapidly to the corresponding large- $\hat{t}$ asymptotes, $\delta \hat{r}_{-}^{2}(\hat{t}) \sim \hat{t}^{1-2 / z}$ and $\delta \hat{r}_{+}^{2}(\hat{t}) \sim \hat{t}^{-2 / z}$. Convergence to the critical asymptote, $\delta \hat{r}_{ \pm}^{2}(\hat{t}) \sim$ const, for $\hat{t} \ll 1$ becomes increasingly better as the critical point is approached.

Deviations from scaling can be rationalized by considering the again universal corrections to scaling. Extending the ansatz, Eq. (1), by an irrelevant parameter $u$ leads to $\delta r^{2}(t ; \varepsilon, u)=t^{2 / z} \Phi_{ \pm}\left(t l^{-z}, u t^{-y}\right)$, where $y$ is a universal exponent. Since $\Phi_{ \pm}$is assumed to be analytic for small arguments, one obtains the leading-order correction upon expanding $\Phi_{ \pm}$to first order in $u$,

$$
\delta r^{2}(t ; \varepsilon)=t^{2 / z} \delta \hat{r}_{ \pm}^{2}(\hat{t})\left[1+t^{-y} \Delta_{ \pm}(\hat{t})\right]
$$

introducing some analytic functions $\Delta_{ \pm}(\hat{t})$. Specializing Eq. (2) to the critical density, i.e., $\hat{t}=0$, yields $\delta r^{2}(t ; \varepsilon=$ $0) \propto t^{2 / z}\left(1+C t^{-y}\right)$, with a single amplitude $C=\Delta_{ \pm}(\hat{t}=$ 0 ); it also identifies $y$ as the leading nonanalytic correction exponent at criticality. Our data for $n_{c}^{*}=0.84$ are compatible with values for $y$ between 0.15 and 0.4 . For the following, we found the choice $y=0.34$ and $C=-0.8$ reasonable, the value for $y$ is supported by theoretical arguments to be presented elsewhere.

Inspection of Fig. 2(b) reveals that corrections to scaling are less relevant for long times, $\hat{t} \gg 1$, whereas significant deviations are visible in the critical regime, $\hat{t} \ll 1$. This observation is consistent with the scaling behavior of the diffusion coefficient and the localization length, see Fig. 3. These findings also suggest approximating the corrections by its value at $\hat{t}=0$, i.e., substituting $\Delta_{ \pm}(\hat{t})=C$ in Eq. (2) for all times, $\delta r^{2}(t ; \varepsilon)=t^{2 / z} \delta \hat{r}_{+}^{2}(\hat{t})\left(1+C t^{-y}\right)$. With $y$ and $C$ already inferred from the data close to criticality, the 


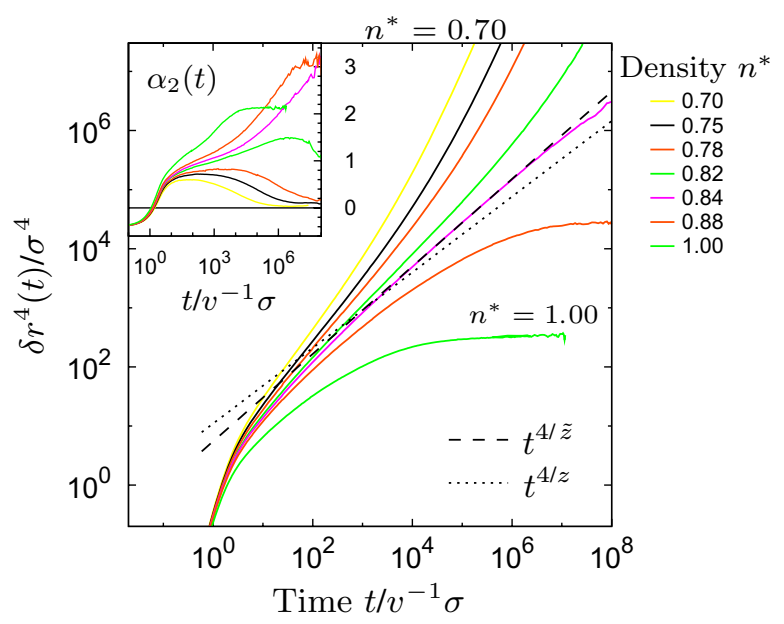

FIG. 4 (color online). MQD $\delta r^{4}(t)$ for densities above and below $n_{c}^{*}$. The dashed and dotted lines compare the two exponents $\tilde{z}$ and $z$. Inset: NGP $\alpha_{2}(t)$ for the same densities; its longtime limit increases as $n_{c}^{*}$ is approached.

correction terms should apply for all densities. Indeed, including this leading-order correction improves the data collapse substantially [Fig. 2(b), right panels].

The presence of two different length scales, $l$ and $\xi$, in the scaling hypothesis, Eq. (1), is not manifested in the MSD; it will, however, affect the higher moments of the probability distribution, e.g., the mean-quartic displacement (MQD), $\delta r^{4}(t ; \varepsilon)=\int d^{d} \mathbf{r} r^{4} G(\mathbf{r}, t ; \varepsilon)$. Above $n_{c}^{*}$, it is easily inferred that the long-time limit of the MQD scales as $\delta r^{4}(t \rightarrow \infty) \sim \xi^{2} l^{2}$. At the critical density, we obtain the long-time asymptote, $\delta r^{4}(t) \sim t^{4 / z}$, with the exponent $z:=(2 \nu-\beta+\mu) /(\nu-\beta / 4) \approx 5.45$ different from $z$. We have evaluated the MQD by our simulation and find agreement with the prediction of continuum percolation at a similar level of significance as for the MSD, see Fig. 4. In particular, for the density $n^{*}=0.84$ the MQD follows a power law with the predicted exponent $\tilde{z}$ for a time window of more than four decades.

A more sensitive quantity is the (first) non-Gaussian parameter (NGP), $\alpha_{2}(t):=\frac{3}{5} \delta r^{4}(t)\left[\delta r^{2}(t)\right]^{-2}-1$, quantifying deviations from a Gaussian distribution [36]. At criticality, it diverges as $\alpha_{2}(t) \sim t^{4 / z-4 / z} \approx t^{0.097}$; direct observation of this very small exponent is expected to be a considerably difficult task. The long-time limits of $\alpha_{2}(t)$ diverge upon approaching $n_{c}^{*}$ from either above or below as $\alpha_{2}(t \rightarrow \infty) \sim|\varepsilon|^{-\beta}$. In particular, the NGP does not vanish in the diffusive regime close to the transition due to the presence of localized particles even below $n_{c}^{*}$. Although there are significant statistical errors in the data for the NGP, Fig. 4 (inset) provides evidence for a significant increase of $\alpha_{2}(t)$ as density approaches $n_{c}^{*}$ from either side. The properties of the NGP demonstrate that the presence of two divergent length scales is crucial for the understanding of the dynamics close to the localization transition.
We are indebted to W. Götze for valuable discussions and critical comments as well as W. Kob and A. Moreno for helpful correspondence.

[1] E. Bertin, J.-P. Bouchaud, and F. Lequeux, Phys. Rev. Lett. 95, 015702 (2005).

[2] L. Berthier et al., Science 310, 1797 (2005).

[3] A. I. Campbell et al., Phys. Rev. Lett. 94, 208301 (2005).

[4] S. Manley et al., Phys. Rev. Lett. 95, 238302 (2005).

[5] E. Zaccarelli et al., Phys. Rev. Lett. 94, 218301 (2005).

[6] B. Ruzicka, L. Zulian, and G. Ruocco, Phys. Rev. Lett. 93, 258301 (2004).

[7] B. J. Alder and T. E. Wainwright, Phys. Rev. A 1, 18 (1970).

[8] J. R. Dorfman and E. G. Cohen, Phys. Rev. Lett. 25, 1257 (1970).

[9] M. H. Ernst, E. H. Hauge, and J. M. J. van Leeuwen, Phys. Lett. A 34, 419 (1971).

[10] K. Kawasaki, Prog. Theor. Phys. 45, 1691 (1971).

[11] D. Bedaux and P. Mazur, Phys. Lett. A 43, 401 (1973).

[12] M. Tokuyama and I. Oppenheim, Physica A (Amsterdam) 94, 501 (1978).

[13] A. Weijland and J.M. J. van Leeuwen, Physica (Amsterdam) 38, 35 (1968).

[14] M. H. Ernst and A. Weijland, Phys. Lett. A 34, 39 (1971).

[15] C. Bruin, Physica (Amsterdam) 72, 261 (1974).

[16] W. Götze, E. Leutheusser, and S. Yip, Phys. Rev. A 23, 2634 (1981); 24, 1008 (1981).

[17] A. Masters and T. Keyes, Phys. Rev. A 26, 2129 (1982).

[18] J. Kertész, J. Phys. Lett. 42, 393 (1981).

[19] W. T. Elam, A. R. Kerstein, and J. J. Rehr, Phys. Rev. Lett. 52, 1516 (1984).

[20] B. I. Halperin, S. Feng, and P. N. Sen, Phys. Rev. Lett. 54, 2391 (1985).

[21] J. Machta and S. M. Moore, Phys. Rev. A 32, 3164 (1985).

[22] O. Stenull and H. Janssen, Phys. Rev. E 64, 056105 (2001).

[23] A. R. Kerstein, J. Phys. A 16, 3071 (1983).

[24] B. Derrida et al., J. Stat. Phys. 36, 31 (1984).

[25] D. B. Gingold and C.J. Lobb, Phys. Rev. B 42, 8220 (1990).

[26] A. B. Harris, S. Kim, and T. C. Lubensky, Phys. Rev. Lett. 53, 743 (1984).

[27] T. C. Lubensky and A.-M. S. Tremblay, Phys. Rev. B 34, 3408 (1986)

[28] For a review see, e.g., S. Havlin and D. Ben-Avraham, Adv. Phys. 51, 187 (2002).

[29] J. Kertész and J. Metzger, J. Phys. A 16, L735 (1983).

[30] This relation may be violated for $|\varepsilon|<0.01$. We checked that our findings are not affected by finite-size effects. A detailed analysis will be presented elsewhere.

[31] This value for $n_{c}^{*}$ corresponds to a critical volume fraction for the obstacles, $\varphi_{c}=1-\exp \left(-\frac{4 \pi}{3} n_{c}^{*}\right)=0.9702(5)$.

[32] M. D. Rintoul, Phys. Rev. E 62, 68 (2000).

[33] D. Stauffer and A. Aharony, Introduction to Percolation Theory (Taylor \& Francis, London, 1994), 2nd ed.

[34] J. P. Straley, J. Phys. C 15, 2343 (1982).

[35] All exponents are calculated consistently based on the values $\beta=0.41, \nu=0.88$, and $\mu^{\text {lat }}=2.0$ [33].

[36] J. P. Boon and S. Yip, Molecular Hydrodynamics (Dover, New York, 1991). 